\title{
Actitudes y percepciones de los docentes universitarios en el proceso de adopción de una innovación: la iniciativa OpenCourseWare en la Universidad de Valencia, España
}

\author{
Attitudes and perceptions of faculty members in the \\ process adopting innovation: The OpenCourseWare \\ initiative at the University of Valencia, Spain
}

Lourdes MARGAIX-FONTESTAD'

Aurora GONZÁLEZ-TERUEL²

Maria Francisca ABAD-GARCÍA²

\section{Resumen}

El proyecto OpenCourseWare, repositorio de recursos educativos en abierto, se inició en la Universidad de Valencia en 2008 y en 2009, sólo incluía 10 asignaturas, ninguna de ellas del área de Ciencias de la Salud. El objetivo de este trabajo es investigar las actitudes de los docentes de esta área así como sus percepciones con relación a este repositorio para determinar cuáles son las estrategias necesarias para extender su uso. Se parte de la teoría de la difusión de las innovaciones de Rogers y de un enfoque cualitativo a través del muestreo teórico, entrevistas semiestructuradas y codificación abierta. Los resultados indican la existencia de dos grandes grupos de profesores. Los adoptantes tempranos con una predisposición alta a participar en la iniciativa y con una percepción positiva de sus atributos y los mainstream o corriente principal más reacios a participar y con una percepción negativa respecto a alguno de sus atributos. La identificación de las características de estos dos grandes grupos marca las líneas de acción y de investigación futura en el ámbito del marketing y difusión, formación, sensibilización y motivación para extender el uso del OpenCourseWare.

Palabras llave: Atributos de la innovación. Categorías de adoptantes. Comportamiento informacional. Difusión de la innovación. OpenCourseWare.

\begin{abstract}
The OpenCourseWare project, an open learning resource repository, was established at the University of Valencia between 2008 and 2009. It initially consisted of only 10 subjects, none of which was in the field of Health Sciences. The objective of this research was to investigate the attitudes and perceptions of faculty members in the field of Health Sciences with regard to the project to identify strategies to increase its use and expand its coverage. We applied Rogers' innovation-diffusion theory along with a qualitative approach using theoretical sampling, semi-structured interviews and open coding. The results indicate the existence of two categories of faculty members. In the first group, we find those who adopt an innovation early, have an inclination to participatein the initiative, and a positive perception of the characteristics of the innovation. In the second, the largest category is composed of those who are more reluctant to participate
\end{abstract}

\footnotetext{
1 Universidad de Valencia, Facultad de Enfermería y Podología, Departamento de Enfermería. Valencia, España.

2 Universidad de Valencia, Facultad de Medicina, Departamento de Historia de la Ciencia y Documentación. Av. Blasco Ibáñez, 15, 46010, Valencia, España. Correspondencia a nombre de/Correspondence to: A. GONZALEZ-TERUEL.E-mail:<agonzal@uv.es>.

Recibido el dia 17/12/2012, re-presentado el 11/6/2013 y aceptado para su publicación el 12/7/2013.
} 
and who have a negative perception of one or more of its characteristics. Identifying the nature of these two groups provides guidelines for further actions and research in marketing and distribution of such projects, training, awareness, and motivation required to increase the use of OpenCourseWare.

Keywords: Innovation attributes. Adopter categories. Information behavior. Innovation diffusion. OpenCourseWare.

\section{Introducción}

El OpenCourseWare (OCW) es uno de los más importantes repositorios de Recursos Educativos en Abierto (OER, Open Educational Resources) promovido en 2001 por el Massachusetts Institute of Technology. Se trata de una publicación digital libre y abierta de materiales educativos universitarios organizados en cursos y que, a menudo, incluyen la planificación y las herramientas de evaluación. El OCW es libre, con licencia abierta y accesible a través de Internet. En los últimos años, las universidades han impulsado el desarrollo de los repositorios OER y en particular del OCW. Por una parte, como extensión del acceso abierto a la información científica, por otra, y en el caso de Europa, en el contexto del desarrollo del Espacio Europeo de Educación Superior (EEES) para la implantación de un modelo pedagógico en el que docentes y estudiantes usen, reutilicen y compartan recursos con el resto de la comunidad educativa (Santos-Hermosa et al., 2012).

Para la implantación del OCW es necesario emplear mucho tiempo y numerosos recursos humanos y materiales. En cambio, los gestores de ese sistema esperan que sea alimentado y utilizado por sus principales usuarios, los docentes. Pero si sólo unos pocos profesores participan en el OCW, su objetivo no se alcanzará pues ellos son el alma mater del sistema. Si esto ocurre, hay una necesidad crítica de entender el porqué (Xu, 2008). En este sentido, la Universidad de Valencia (UV) se incorporó al proyecto en 2008, al año siguiente se incluyeron los primeros materiales docentes y en 2009, el momento de realizar la presente investigación, sólo incluía 10 asignaturas, ninguna de ellas del área de ciencias de la salud.

La mayor parte de investigación publicada sobre la implantación de los OER está centrada en los aspectos técnicos y organizacionales y en menor medida en la dimensión individual y social de este proceso (SantosHermosa et al., 2012). En este sentido, se destacan los trabajos de Shea et al. (2006) sobre las actitudes que condicionaban la adopción del repositorio Multimedia Educational Resource for Learning and Online Teaching (MERLOT), el trabajo de Gadd et al. (2007) sobre las actitudes de profesores hacia la propiedad de derechos de autor de sus materiales o el estudio de Rolfe (2012) sobre las actitudes del personal universitario en el área de Ciencias de la Salud y de la Vida hacia el contenido en abierto, entre otros.

En este contexto, esta investigación plantea como objetivos conocer las actitudes hacia el OCW de los docentes de Ciencias de la Salud de la UV e investigar cómo perciben esta herramienta con relación a su integración en el proceso de enseñanza-aprendizaje, todo ello con el fin de diseñar estrategias que impulsen su uso. Como marco de referencia se parte de la teoría de la difusión de las innovaciones de Rogers (2003), considerando el OCW como una innovación tecnológica y educativa pero también informativa en la que los docentes son los principales usuarios, generando los contenidos para compartirlos posteriormente.

\section{La difusión de las innovaciones}

La implantación del OpenCourseWare en la Universidad de Valencia es un claro ejemplo de introducción de una innovación en un contexto con una larga tradición de enseñanza presencial. Esta innovación puede investigarse en su vertiente tecnológica pero también desde el punto de vista individual y social. En este sentido, las teorías sobre la difusión de las innovaciones proporcionan una base conceptual para el estudio de las dimensiones que intervienen en ese proceso y un procedimiento para favorecer su adopción. Como señala Rogers (2003) dichas teorías, al igual que las de aprendizaje social, tratan de explicar cómo y por qué los individuos cambian su conducta a través de la comunicación con otras personas, siendo el intercambio de información un aspecto esencial para que se produzca este cambio y los vínculos de una red como su principal explicación. 
La asunción de este enfoque teórico y, en concreto, determinados aspectos de la teoría de la difusión de las innovaciones de Rogers (2003), se consideró el más adecuado para estudiar los potenciales usuarios del OCW por dos razones. Primero, porque permitía centrar la investigación en sus características como individuos de un grupo social asumiendo un rol en el proceso de difusión de la innovación. En segundo lugar, porque permitía sistematizar sus actitudes y por tanto determinar sobre qué aspectos era necesario incidir en ese proceso. En el contexto del estudio de las actitudes hacia los OER, esta teoría sirvió de fundamento teórico en los trabajos de Shea et al. (2006), Xu (2008) y Arendt y Shelton (2009), entre otros.

Según esta teoría, la difusión es “Un proceso por el cual una innovación se comunica por determinados canales a través del tiempo entre individuos de un sistema social" (Rogers, 2003, p.11). Las dimensiones que intervienen en este proceso son: canales de comunicación, tiempo, percepción de la innovación y sistema social.

Para alcanzar los objetivos propuestos, se utilizó la dimensión tiempo así como la percepción de las características de la innovación por parte de los docentes. Así, para Rogers (2003) el tiempo durante el cual una innovación se difunde determina la existencia de cinco categorías de adoptantes según su ritmo previsible de adopción: a) innovadores que importan la innovación incorporándola al sistema asumiendo un papel fundamental en su difusión; b) primeros adoptantes que aceptan la innovación antes que la mayoría pero más prudentemente asumiendo el liderazgo entre sus colegas; c) mayoría precoz que necesita más tiempo para adoptar la innovación y legitima la innovación; d) mayoría tardía que adopta las nuevas ideas por presión del entorno necesitando mayor motivación y e) tradicionales que tienen como referencia el pasado aceptando la innovación con reservas.

Por otra parte, Rogers (2003), considera que lo importante es la percepción subjetiva que los adoptantes potenciales tienen de la innovación, para lo cual define cinco atributos: a) ventaja relativa o la percepción de que una innovación es mejor que la idea a la que reemplaza; b) compatibilidad con el sistema existente; c) complejidad o dificultad para entender y utilizar la innovación; d) experimentación o el grado en que una innovación puede ser experimentada y e) visibilidad de los resultados de la innovación. Así, las innovaciones que son percibidas con una mayor ventaja relativa, compatibilidad, experimentación y visibilidad y menor complejidad se adoptarán con mayor rapidez.

\section{Métodos}

La presente investigación se ha diseñado como un estudio exploratorio y adopta un enfoque metodológico cualitativo. Dicho enfoque se considera el más adecuado para abordar aspectos poco estudiados en la implantación del OCW, como son la dimensión individual y social de este proceso. Además, se considera que aporta mayor flexibilidad a la hora de observar el objeto de estudio y una mayor profundidad y detalle en el registro y análisis de los aspectos observados.

La población en la que se centra la investigación es la de los docentes del área de Ciencias de la Salud de la UV. Concretamente los docentes de Enfermería, Podología y Fisioterapia, titulaciones que por la implantación del EEES, aumentaron los créditos de docencia pasando de diplomatura (3 años) a grado (4 años). Para la selección de los informantes, se utilizó un muestreo teórico (Glaser \& Strauss, 1967) en el que ni el número ni sus características estaban determinadas de antemano. Así, el proceso de recogida y análisis de la información transcurrió simultáneamente. La recogida de datos se inició con los directores de los centros y departamentos así como con aquellos individuos que respondieron al Ilamamiento de colaboración. Tras el primer análisis de sus respuestas, siguió una estrategia de bola de nieve en la que la localización de nuevos informantes se hizo por indicación de los primeros (Patton, 2002). La selección de más informantes finalizó cuando se alcanzó la saturación teórica y la información aportada no añadía ninguna idea, opinión o actitud nueva. En total se realizaron 26 entrevistas. La Tabla 1 resume las características de los entrevistados, representados por un código (p.e. E01) que sirve para ilustrar los resultados expuestos en el siguiente epígrafe.

Para la obtención de información se administraron entrevistas semiestructuradas guiadas por un guión previo de cuestiones relacionadas con los objetivos. 
Inicialmente se solicitó autorización institucional y, antes de comenzar cada entrevista, los informantes leían y firmaban un consentimiento informado.

Las entrevistas se realizaron entre los meses de febrero y junio de 2009, tuvieron una duración media de 20 minutos, fueron registradas en soporte digital y almacenadas posteriormente a través del software atlas.ti5.0.

Para el análisis de los datos, se escuchó el contenido de las respuestas identificando y transcribiendo los fragmentos más significativos. Esta información se analizó por medio de un proceso de codificación abierta para, posteriormente, comparar los códigos resultantes con las categorías de adoptantes y los atributos de la innovación de Rogers (2003). La asignación de una categoría de adoptante a cada uno de los informantes estuvo guiada principalmente por su predisposición manifiesta a adherirse a la iniciativa OCW y su destreza con el uso de la tecnología. Con relación a la experimentación como atributo de una innovación, dado que el sistema no se podía probar si el docente no incluía sus materiales, se consideró de acuerdo con su predisposición a experimentar tal innovación. Con el fin de asegurar la confiabilidad de la investigación principalmente se aplicaron dos de las estrategias propuestas por Lincoln y Guba (1985). En concreto, la confirmación por parte de algunos informantes de los resultados de las entrevistas y la descripción densa.

\section{Resultados}

Los resultados se exponen siguiendo las categorías de adoptantes de Rogers respecto a la adopción potencial de la iniciativa OCWy, para cada tipo de adoptantes, según las percepciones que tuvieran respecto a esta innovación. La Tabla 2 resume los resultados obtenidos.

Es necesario mencionar que, de los 26 incidentes analizados, ninguno pudo clasificarse como innovador pues, aunque el OCW estaba un año ya en funcionamiento, ninguno de ellos había participado todavía.

a) Primeros adoptantes: los cuatro profesores clasificados como primeros adoptantes se mostraron favorables a la adopción inmediata de la iniciativa OCW. Todos ellos tenían dominio de la tecnología, gran experiencia y su plaza docente consolidada. Además, los materiales de sus asignaturas ya los tenían disponibles a través de la red o en el pasado los habían tenido.

Opinaron que el OCW podía favorecer el autoaprendizaje del alumnado en el marco del EEES, aumentar la calidad docente de los materiales por el

Tabla 1. Características de los entrevistados.

\begin{tabular}{llll}
\hline \multirow{2}{*}{ Experiencia docente } & \multicolumn{2}{c}{ Vinculación laboral } \\
\cline { 2 - 3 } & \multicolumn{1}{c}{ Contratado } & \multicolumn{1}{c}{ Investigador } & \\
\hline 1 a 2 años & E03, E05, E14, E23 & E04 \\
3 a 5 años & E15, E20, E26 & E16, E17, E21 & \\
$\begin{array}{l}\text { 6 a 10 años } \\
\text { Más de 15 años }\end{array}$ & & E067, E08, E09, E12, E18, E22, E13, E24, E01, E02, E10, E19, E25, \\
\hline
\end{tabular}

Fuente: Elaboracion propia (2012).

Tabla 2. Número de profesores clasificados en cada categoría de adoptantes y percepción de los atributos de dicha innovación según la teoría Rogers (2003).

\begin{tabular}{|c|c|c|c|c|c|c|}
\hline Categorías de adaptantes & Profesores (n) & Ventaja relativa & Compatibilidad & Complejidad & Experimentación & Visibilidad \\
\hline Primeros adoptantes & 4 & Sí & Sí & Baja & Sí & Sí \\
\hline Mayoría precoz & 4 & Sí & Sí & Baja & Sí & Sí \\
\hline Mayoría tardía & 15 & Sí/No & Sí & Alta/baja & No & No \\
\hline Tradicionales & 3 & No & Sí & Alta & No & No \\
\hline
\end{tabular}

Fuente: Elaboracion propia (2012). 
aumento de la competitividad y la exposición pública de los mismos, así como la satisfacción personal por el trabajo bien hecho: Te expones a otros profesores, a otras universidades, y esto, si tú tienes un poco de amor propio e interés, pues claro, ya no es para un grupo reducido [... . sino que aquí ya te expones a un mayor número de gente, y eso es una gran responsabilidad [E02].

Una actitud de predisposición a participar en el OCW se traduce en una percepción de esta iniciativa como ventajosa por su contribución a la generación de conocimiento y junto con ello, compartir el material docente se vio como un mecanismo para garantizar la propiedad intelectual:

\begin{abstract}
Algunos ya se han quejado de ver su material publicado por allí, en el Rincón del vago [...] parte de los apuntes de clase, y bueno algún otro apropiarse de ello sin consentimiento, no?. Eso alguna profesora ya se ha quejado y tal vez, el OpenCourse pueda garantizar, como mínimo cita al profesor que ha elaborado ese material [E09].
\end{abstract}

Igualmente los primeros adoptantes vieron la iniciativa compatible con el sistema previamente utilizado (aula virtual) incluso considerando la posibilidad de interconexión entre los dos sistemas. No consideraron el sistema como complejo, tanto es así que alguno de ellos no esperaba a la experimentación (probar el nuevo sistema). No obstante, mostraron reservas al considerar que para muchos de sus compañeros su adopción se percibiría como compleja. Por último, en cuanto a la visibilidad, consideraban que contribuiría a aumentar el prestigio de la universidad en general y de la escuela en particular: Si los profesores hacemos eso y lo hacemos bien, aumentarán las consideraciones de la escuela cara al exterior. De la escuela y de la universidad. Yo creo que es un prestigio para la universidad [E02].

b) Mayoría precoz: como mayoría precoz, se clasificaron cuatro docentes que mostraron su interés por participar en el OCW pero no de forma inmediata sino tras un tiempo para la revisión de los materiales. Todos ellos tenían en común su experiencia docente así como el puesto de trabajo consolidado (profesor titular). Con relación a su destreza con la tecnología, todos menos uno, no mostraron problemas. Algunas opiniones que emitieron, más allá de las opiniones de los primeros adoptantes, fueron la posibilidad de una mejor coordinación con los demás compañeros, interacción con los docentes de otras universidades y posibilidad de aumentar la eficacia y economía de la difusión de su trabajo:

En principio, yo síque estaría de acuerdo en colgar las dos asignaturas y en facilitar no sólo el programa y la bibliografía sino especialmente los contenidos, porque obedece a un trabajo personal del profesory que tendría una mayor trascendencia y una mayor difusión de la información que por otros mecanismos sería mucho más costosa [E07].

Tal y como sucedía con el grupo de primeros adoptantes, los docentes del grupo de mayoría precoz consideraron la iniciativa con gran ventaja relativa, compatible con el sistema existente y no la percibieron como compleja. Igualmente consideraron que la innovación sí sería compleja para algunos compañeros:

Aquí hay muchos compañeros que, bueno, por diversas circunstancias, por la edad, porque les ha cogido a contrapié todo el tema de la informática [...],yentonces están encasillados ahí, en un lugar, donde no moverán pieza. Terminarán su ciclo laboral sin acogerse a ésta ni a otra plataforma [E12].

Los docentes de este grupo percibieron la iniciativa como experimentable y estarían dispuestos a probarla, además como ejemplo para el resto de compañeros:

Yo estaría dispuesto a participar en una prueba de este tipo, porque, efectivamente aunque yo creo que, no creo que tenga dificultad, por lo tanto, la prueba debe salir bien, pero bien, a mí, yo creo que sería interesante probarla sobre todo para demostrar a personas que no estén del todo convencidas de la misma [E10].

Por último, en cuanto a la visibilidad, también encontraron que la adhesión a la iniciativa sería visible para el resto de los compañeros. No obstante, uno de los informantes apuntó la posibilidad de sufrir críticas destructivas por parte de ellos.

c) Mayoría tardia: en esta categoría se clasificaron la mayor parte de profesores con 15 casos. Eran docentes que previsiblemente se incorporarían más tarde a la iniciativa porque expresamente informaron de que no querían o no podían hacerlo. Su destreza con el uso de la tecnología era suficiente, excepto en un caso. Respecto al tipo de vinculación con la universidad, ésta fue variable. 
En general, no dudaron de que la iniciativa llevara a una mejora de los materiales docentes, mejorara la coordinación con el resto de profesores y permitiera al alumnado el acceso fluido a sus materiales, entre otras. Sin embargo, esta opinión positiva estaba supeditada al reconocimiento de la propiedad de dichos materiales: Yo no tengo ningún problema siempre que la gente referencie. Yo cuando he citado algo que está abierto lo he referenciado. Supongo que es una forma de respeto unos a otros [E05].

Frente a estas opiniones positivas, en este grupo, se opinó de forma negativa en aspectos como la relación del esfuerzo que supondría la participación en el OCW frente al beneficio que obtendrían por ello: Para qué quiero colgarlo yo ahí si no me gano nada, ya no económicamente, sino, no sé, yo prefiero publicar en un libro de papel, no porque sea de papel, sino [...], o en una revista científica [E21].

Otras opiniones negativas respecto al OCW tuvieron que ver con la falta de tiempo por ejercer cargos de gestión o por la necesidad de revisión de los materiales. A este respecto, se vislumbra una preocupación sobre la calidad de los materiales en dos sentidos. Por una parte por la dudosa fiabilidad de la información depositada en el OCW por no estar sometida a revisión: El hecho de que un profesor cuelgue sus apuntes y no haya [...] nadie que los haya revisado, sabes lo que te digo, a mí no me da ninguna fiabilidad, y más en ciencias de la salud [E21].

Por otra parte, por la inseguridad de poner en abierto materiales que no han sido revisados por otros: Yo necesito formación para mejorar mis clases, para mejorar los contenidos de mi asignatura, yo no me veo capacitada para poner unos apuntes en la red [E21].

También otras opiniones negativas estaban relacionadas con la obligatoriedad de incorporar una asignatura completa o por considerar que sería una medida que favorecería el absentismo del alumnado.

Respecto a la percepción de la innovación, todos los informantes consideraban la iniciativa con ventaja relativa condicionada al respeto de la propiedad de los materiales, tal y como se ha descrito antes, y compatible con el sistema anterior. Sin embargo, con respecto a los otros atributos la percepción variaba. Con relación a la complejidad, algunos informantes consideraron fácil su participación en el OCW o preferían probarlo antes de emitir un juicio. Sin embargo, otros la consideraron compleja por la necesidad de incluir asignaturas completas cuando sólo eran responsables de parte de esa asignatura, por la falta de destreza con la tecnología, por la falta de un buen material docente y las dudas relacionadas con la inexistencia de mecanismos de revisión de su contenido o por las características de una materia particular: Porque hay que tocar, la fisioterapia uno debe saber la presión que debe ejercer con las manos, la temperatura que está una articulación cuando la toca, esto por Internet es imposible [E17].

Respecto a la experimentación, no mostraron voluntad de experimentar por falta de tiempo, por no tener el temario completo o por tener poca experiencia con la asignatura. Por último, consideraron la iniciativa como no visible y sólo se podría considerar visible si el profesorado tuviera interés en la iniciativa, aunque para ello habría que difundirla más.

d) Tradicionales: en esta categoría se incluyeron tres profesores que, aunque pudieran percibir alguna ventaja, no estaban dispuestos a participar en el OCW. Todos ellos tenían destreza deficiente en el uso de la tecnología y sus materiales docentes o no los distribuían o lo hacían a través de fotocopias.

Con relación al carácter de su plaza docente se dieron dos casos. Por una parte, uno con escasa experiencia y plaza no consolidada, reticente a la adopción del OCW y en general a la distribución de los materiales por él elaborados:

Hombre a míme cuesta, eh, a míme cuesta, y me cuesta a veces darles a los alumnos, cuando hay toda una tarea, según el tipo de materiales que tú entregas, no?, yo nunca los doy [...] nunca les doy antes de una clase, porque quiero que me estén escuchando, no quiero que tengan los apuntes [E15].

Y porque consideraban que eran materiales especializados que no deberían estar accesible al público general: La información que tiene que recibir el público no es la información del material que compartimos los profesionales, por eso ellos no son profesionales y nosotros sí. No podemos bajar tanto, tanto, tanto [E15].

Por otra parte, dos docentes profesores titulares con gran experiencia docente, cercanos a la jubilación 
que opinaban que eran propietarios de la información y que se trataba de iniciativas para jóvenes: A míme parece muy bien, me parece un avance, pero como te decía antes, no soy partidario de nuevas [...] dejo estas iniciativas a los que vengan detrás de mí [E19].

Con relación a los atributos de la innovación, al contrario que las categorías de adoptantes anteriores, la percepción fue menos favorable a su adopción. Por una parte no percibían la ventaja relativa pues no consideraban la iniciativa como algo distinto al sistema de aula virtual ya utilizado en la universidad.

Respecto a la compatibilidad, todos los informantes percibieron la iniciativa como compatible con el sistema actual. Sin embargo, los docentes de este grupo percibieron gran complejidad porque era necesario utilizar la tecnología para disponer de un material docente adecuado: Yo aquí ahora como estoy lo veo difícil pero no por el método, sino por mi incapacidad, porque yo no soy un experto en sistema informático y todo eso, en fin, yo de informática justita [E22].

Finalmente, los docentes de este grupo opinaron que no les interesaría probar la iniciativa y, en cuanto a la visibilidad, consideraron que el resto de sus compañeros ni siquiera mirarían los materiales disponibles en la plataforma OCW.

\section{Discusión}

La adopción de un enfoque cualitativo, en el marco de la teoría de Rogers (2003), para el estudio de las actitudes y percepciones del OCW por parte de los docentes de la UV, ha resultado de utilidad por diversos motivos.

En primer lugar, respecto a la metodología, las entrevistas semiestructuradas han permitido obtener información de gran riqueza gracias a la expresión libre de cada informante. En este sentido, son reveladores los resultados de Shea et al. (2006) que, aunque partiendo del mismo marco teórico, fueron limitados por la imposibilidad de indagar más allá de las contestaciones precisas de un cuestionario estructurado.

En segundo lugar, utilizar las categorías de adoptantes de una innovación de Rogers (2003) como criterio de segmentación de los potenciales usuarios del
OCW ha permitido alejarse de categorizaciones basadas exclusivamente en criterios socio-demográficos que, al menos en una fase exploratoria, podría alejar del verdadero foco de interés que es conocer las dimensiones que intervienen en el objeto de estudio. Además, ha permitido detectar dos grandes grupos de docentes según su predisposición a participar en el OCW, cuyas características y necesidades diferenciadas determinan el diseño de programas incentivadores distintos. Así, para el contexto estudiado, al igual que Zayim et al. (2006), sería posible reconocer a un primer grupo de adoptantes tempranos (primeros adoptantes y mayoría precoz) y un segundo grupo mainstream o corriente principal (mayoría tardía y tradicionales). En el primer caso, se trata de docentes con una predisposición alta a participar en la iniciativa que requerirían programas que potenciaran su papel como líderes de opinión y por lo tanto difusores de la innovación, en la línea de lo que Rogers (2003) denomina agentes de cambio. Y en el segundo, docentes más reacios a participar en el OCW, cuyos programas deberían orientarse a la sensibilización, formación y al fomento de la autoeficacia.

A la vista de los resultados, en primer lugar llama la atención las reservas de algunos informantes, de cualquiera de los grupos antes mencionados, respecto a que muchos compañeros participen en el OCW e, incluso, de que la universidad sea capaz de implantar con éxito esa iniciativa. Estas opiniones podrían responder a la falta de un clima institucional favorable a la adopción del OCW que requeriría, tal y como plantea Margaryan et al. (2006), el que formara parte de una estrategia organizacional a largo plazo, visible e identificable para todos los miembros. Y que ésta no fuera percibida como una iniciativa aislada motivada por las presiones del entorno.

Junto con este clima desfavorable, a la vista de los resultados, existen dos factores como son la experiencia y la estabilidad de la plaza docente que parecen condicionar la actitud hacia la aceptación de una innovación. En este sentido los más propensos a la adopción del OCW son personas con una gran experiencia docente junto con una plaza más estable. Correspondería con lo que Sikes (1985) denomina tercera etapa o de asentamiento, en la que hay un período de gran capacidad física e intelectual. Pero a la vez, esta experiencia junto con una gran estabilidad en personas 
próximas a la jubilación también es determinante de un mayor recelo a la adopción de esa innovación. Así, las personas más reacias a participar en el OCW han sido docentes próximos a la jubilación, lo que Huberman (1989) denomina desencantados, que lo consideran una buena idea pero para los más jóvenes. Sin embargo, algunos docentes jóvenes también pueden mostrarse reacios por no encontrar beneficios curriculares. En este caso, el puro altruismo al que Arendt y Shelton (2009) se refieren como una de las más importantes motivaciones, está a mucha distancia de esta realidad.

Al examinar los resultados de la percepción de los atributos de la innovación, igualmente se detectan dos grandes grupos que coinciden con las categorías de adoptantes según su predisposición a participar en el OCW antes mencionadas. Por una parte, los adoptantes tempranos que lo perciben con gran ventaja relativa compatible, experimentable, visible y poco complejo, o sea, la situación ideal para Rogers (2003), por otra, está el grupo de los mainstream que perciben su ventaja relativa sólo parcialmente, cierta complejidad y lo consideran no experimentable ni visible.

Por lo tanto, tal y como antes se ha mencionado, las personas consideradas como adoptantes tempranos y que tienen mejores opiniones respecto a los atributos de la innovación deben ser consideradas personas clave para impulsar su difusión. En este sentido, Martins et al. (2004) considera que éstos deben participar en programas de fomento de la innovación que versen sobre sus ventajas y usos, con especial hincapié en la visibilidad y experimentación. Pero además Xu (2008) considera que los cargos institucionales deben asumir también el papel de promotores de la iniciativa para ganar la confianza del resto de los adoptantes. Sin embargo, en el contexto investigado, los individuos con cargos de gestión entrevistados se refirieron a la falta de tiempo derivada de dicho cargo como el motivo o barrera para no participar de forma inmediata.

Respecto al grupo de docentes con percepciones poco favorables a la adopción del OCW, de entre todos los atributos investigados, la ventaja relativa y la complejidad podrían considerarse críticas para la adopción del OCW. Respecto a la ventaja relativa, mientras que el grupo de tradicionales no percibe esa ventaja quizás por desconocimiento de la filosofía del
OCW, en la mayoría precoz sí se percibe pero siempre que exista reconocimiento de la propiedad intelectual. Si un profesor considera que la ventaja de compartir es el beneficio de otros, sin el reconocimiento de su trabajo y sin incentivos suficientes, difícilmente participará en una iniciativa como el OCW. Con relación a la complejidad, aparece la idea de la falta de control de la calidad de los materiales docentes y por lo tanto la inseguridad de que sea adecuado exponerlos al escrutinio público. Tal y como afirman Heery y Powell (2006), los docentes tradicionalmente han tenido el control sobre sus materiales e incluirlos en cualquier tipo de repositorio les plantea dudas respecto a la propiedad, los derechos de autor, el control de calidad, el intercambio y la reciprocidad de tal intercambio. Por otra parte, ambas cuestiones, tanto la propiedad intelectual como el control de la calidad de los materiales son inquietudes comunes que surgen en la investigación de las actitudes de los docentes hacia los OER. En este sentido, Rolfe (2012) considera que la institución debe establecer políticas claras y transparentes tanto en el ámbito de la asistencia y orientación como en el de la gestión de los derechos de autor. Mientras que Margaryan et al. (2006) consideran que una política institucional debe establecer procedimientos para incentivar la participación de los docentes incluyendo un sistema de revisión de los materiales que, además de mejorar la percepción del sistema respecto a su complejidad, podría ser un mecanismo que impulse su participación. Como afirma Ashetal.(2001), para un sistema percibido extremadamente complejo, seria ideal que se implementara con la máxima participación de los usuarios, con altos niveles de apoyo y estar rodeado de una atmósfera de confianza y colaboración.

\section{Conclusión}

Conocer los factores que condicionan el uso del OCW de la Universidad de Valencia es fundamental para su gestión, más en tiempos de restricciones económicas. La implicación y el compromiso del profesorado en esta iniciativa son de vital importancia para asegurar su utilidad y pervivencia. La investigación de las actitudes y percepciones de los docentes ha permitido obtener una 
visión amplia de los aspectos críticos que pueden dificultar o favorecer su uso. Los resultados obtenidos marcan diversas líneas de acción para una respuesta inmediata en cuanto al fomento del uso del OCW pero también líneas de investigación que deben desarrollarse en un futuro en el ámbito del marketing y difusión, formación, sensibilización y motivación.

\section{Referencias}

Arendt, A.M.; Shelton, B.E. Incentives and disincentives for the use of OpenCourseWare. International Review of Research in Open and Distance Learning, v.10, n.5, p.1-25, 2009.

Ash, J.S. et al. A diffusion of innovations models of physician order entry. Proceedings AMIA Symposium, p.22-26, 2001 (PMID: 11825150).

Gadd, E. et al. A comparison of academics' attitudes towards the rights protection of their research and teaching materials. Journal of Information Science, v.33, n.6, p.686-701, 2007.

Glaser, B.; Strauss, A.L. The discovery of grounded theory: Strategies for qualitative research. New Brunswick: Aldine Transaction, 1967.

Heery, R.; Powell, A. Digital repositories roadmap: Looking forward. London: Jaint Information Systems Committee, 2006. Available from: <http://www.jisc.ac.uk/uploaded_documents/ rep-roadmap-v15.doc>. Cited: Dec. 12, 2012.

Huberman, M. The professional life cycle of teachers. Teachers College Record, v.91, n.1, p.31-57, 1989.

Lincoln, Y.; Guba, E. Naturalistic inquiry. Newbury Park: Sage, 1985.

Margaryan, A. et al. CD-LOR delirelable 1: Report on learning communities and repositories. Glasgow: Centre for Academic Practice and Learning Enhancement, 2006. Available from: <http://www.academy.gcal.ac.uk/cd-lor/learning communitiesreport.pdf>. Cited: Dec. 12, 2012.
Agradecimientos

Esta investigación se ha realizado en el marco del proyecto "El acceso abierto a la producción científica (open access) en España: análisis del grado de implantación y de la sostenibilidad de un nuevo modelo de comunicación científica", financiado por el Gobierno de España. Ministerio de Ciencia e Innovación. Plan Nacional de I+D (CSO2011-29503-C02-02).

Martins, C.B.M.J. et al. Factors influencing the adoption of the internet as a teaching tool at foreign language schools. Computers \& Education, v.42, n.4, p.353-374, 2004.

Patton, M.Q. Qualitative research and evaluation methods. $3^{\text {th }}$. Thousand Oaks: Sage, 2002.

Rogers, E. Diffusion of innovations. $5^{\text {th }}$ ed. New York: Free Press, 2003.

Rolfe, V. Open educational resources: Staff attitudes and awareness. Research in Learning Technology, v.20, n.1, p.1-13, 2012.

Santos-Hermosa, G. et al. Recursos educativos abiertos: repositorios y uso. El Profesional de la Información, v.21, n.2, p.136-145, 2012.

Shea, P. et al. Adoption of the multimedia educational resource for learning and online teaching (MERLOT) among higher education faculty: Evidence from the state University of New York. Journal of Online Learning and Teaching, v.2, n.3, p.136-156, 2006.

Sikes, P. The life cycle of the teacher. In: Ball, S.J.; Goodson, I.F. (Ed.). Teachers' lives and careers. London: Falmer Press, 1985. p.27-60.

$\mathrm{Xu}, \mathrm{H}$. The theory analysis of faculty participation in institutional repositories. Occasional Paper Series, n.1, p.3-15, 2008.

Zayim, N. et al. Technology adoption of medical faculty in teaching: Differentiating factors in adopter categories. Educational Technology \& Society, v.9, n.2, p.213-222, 2006. 

